\title{
Production pilot for co-operation in factory development
}

\author{
Chen, D. Kjellberg, T. Svensson, R. Sivard, G. \\ Computer Systems for Design and Manufacturing, Production Engineering, KTH - Royal Institute of Technology \\ Brinellvägen 68, Stockholm, 100 44, Sweden, Tel. +46-7909491 \\ E-mail: danfang.chen@iip.kth.se \\ E-mails: tk@iip.kth.se; gunilla.sivard@iip.kth.se; rsv@iip.kth.se
}

\begin{abstract}
This paper is dedicated to the factory development domain where management of established knowledge and company practice is a big challenge because its process involves many domains such as process planning, production investment and building construction, etc. This article presents an in-house developed production pilot for a better cooperation in factory development. Details about methodologies and the thinking behind the pilot will be presented in the paper. The pilot aims to ease the knowledge sharing and reuse, create a value-adding working process, and ease the collaboration and semantic interpretation between disciplines within factory development.
\end{abstract}

Keywords:

Production pilot, Factory development, Knowledge management

\section{INTRODUCTION}

Value creation is the goal of every company and a good way to create value is combining knowledge and people together with technology. Knowledge sharing is one of the big issues in today's companies, due to several reasons, such as the life style change in today's society where people change jobs more frequently. This brings forth the concept knowledge sharing, due to the change of "work-model". Knowledge needs to be shared, reused and passed forward much faster than before.

There are many ways to share knowledge. The internet is the latest and largest source of knowledge and information. The libraries are not always the first choice anymore. There are just as many people trying to get the knowledge and information from the internet as there are people trying to share their knowledge and information on the internet. The internet is however not an organized knowledge sharing system, even if today's search engines, with their power in finding search terms, may give that appearance. Apart from using the internet, another common method to get information and knowledge is to ask friends or colleagues around oneself, in order to get a task done.

Factory development is a complex process and involves many disciplines and people (see section 5.3). An integrated knowledge sharing method will provide the industry with a shortened project time, avoid mistakes caused by misunderstanding of each other etc.

This paper presents a production pilot which can be seen as a model-based knowledge reference system for co-operation in factory development, both within a company and between companies. This work is an attempt to:

- Ease the knowledge and information sharing and reuse, in factory development.

- Ease the collaboration and semantic interpretation between disciplines within factory development.

\section{METHOD}

It is a huge job to develop a pilot that is on same level as a model- based knowledge reference system and it is essential to break this development into two parts, the production pilot as itself and a modeling application to build this pilot.

For the pilot, the most essential part is the one concerned with knowledge and information related to factory development, where reference material has been gathered from industry and academy for more than three years in the research project ModArt (model driven part manufacturing) [1]

To handle all the information and knowledge that has been gathered, information management is essential and many questions need to be answered, particularly this question:

How should information and knowledge be represented, applied and managed in this pilot for the best utilization?

With many questions in mind, the knowledge and information have been sorted out in two levels for the best representation in the pilot. One level is the model-level, which has been modeled according to the SADT principle [2]. The other level is the documented advice in the model. This separation makes the knowledge and information explicit and easy to update. More details are described later on in this paper.

\section{KNOWLEDGE DEFINITIONS \\ 3.1 Existing definitions}

When talking about knowledge, the differences between data, information and knowledge need to be discussed first. Information is data in a context and knowledge is the ability to interpret information in the right context. Competence is then practical ability to perform [3]. Only with the right competence can the knowledge then form the basis for a good decision or action to take.

According to Wei He et al., Gray and Meister define knowledge as "a mix of expertise, experience, process, conceptual information and insights that provides a framework for decision-making or problem-solving" [4]. Sean Cochrane et al. states that the information exists when the relationships between data are recognized within a specific context and the knowledge is 
information with added detail relating to how it should be used or applied [5].

Knowledge can be divided into levels, one example is organizational knowledge. P.H. Hendriks has pointed out that there are three levels of organizational knowledge; strategic, tactical and operational. The knowledge in the pilot is covered by all these three levels. The knowledge also has a value chain of six stages which are crucial in knowledge management. These stages are the assessment of current knowledge, the establishment of required knowledge, the development of new knowledge, the distribution and sharing of knowledge, the application of knowledge, and the maintenance and evaluation of knowledge [6].

\subsection{Use SADT modeling principle to represent knowledge}

In the pilot, knowledge is divided into two levels as mentioned in the earlier chapter. The SADT modeling principle is used to represent and analyze the model level of the knowledge. The rules/principles of the SADT will point out how information should be used, which is in line with the knowledge definition of Sean Cochrane et al. The activity with ICOM (input, control, output, mechanism) [2] is the basic principle of SADT. By structuring the information in the activity model with ICOMs, the information will be more complete and the singularity in the information flow will be minimized. This means in the factory development process, information in the ICOMs for each activity needs to be fulfilled before moving to the next activity.

\section{THE MODELING APPLICATION}

The modeling application is a must for realizing the pilot. It is here in the modeling application the SADT rules are embedded. During the development of the modeling application the focus has been around its benefit for the users and how to realize the model-based idea. Benefits of the digital approach are that the information can be cross-linked and that users are always presented with the most recent information, which they can edit and improve at any time. The cross-linking provides an extra layer of understanding by enabling the user to see the information from different perspectives, which can only be achieved in a reasonable way using a digital solution.

A backing idea of the modeling application is easy access and easy understanding. In support of the easy access, the only software the user needs in order to access and use the modeling application is a basic web browser, since all the information is presented as ordinary HTML, styled with CSS. The use of more advanced web technologies is kept on a very low level, JavaScript is only used sparsely and Flash is not used at all. In fact, a user with complete lack of JavaScript can still view all of the information that form the contents of the modeling application, albeit a little less visually appealing. The benefits of these non-advanced web technologies in terms of ease are both that the data load is reduced, which in turn makes the information faster to load, but also that the requirements of the viewer application are kept on a low level. Thanks to this, using the modeling application is not limited to powerful desktop computers equipped with advanced browsers and high bandwidth connections but also enables weaker systems to gain access. This makes it possible to use the modeling application on a small portable device, such as a surf tablet or a smart phone, which can be carried around on the factory floor when e.g. performing maintenance.

It is only when editing the contents of the modeling application that JavaScript is really required. In order to input new information, or edit already existing information, the user is presented with an editing interface, illustrated in Figure 1. Figure 1 is for a general understanding of the modeling application and not for detail evaluation, therefore the details in Figure 1 are not readable.

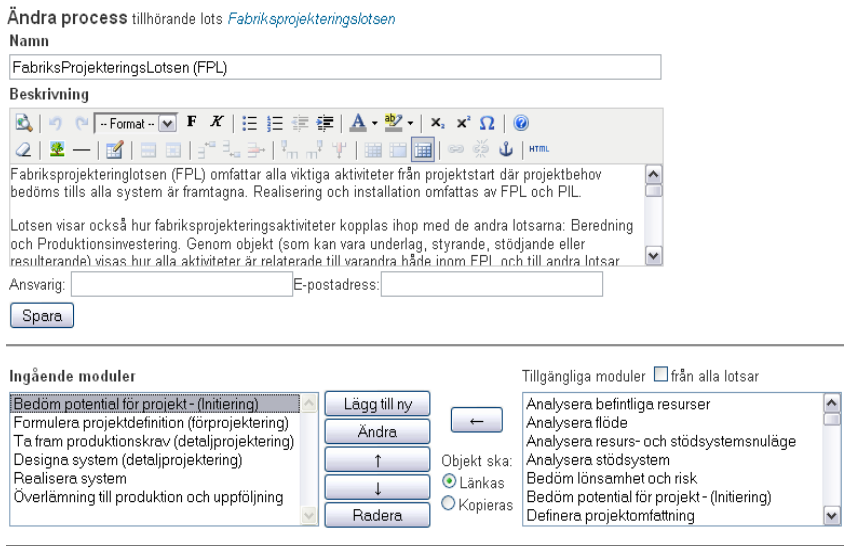

Redigera förälder | Gå till processen

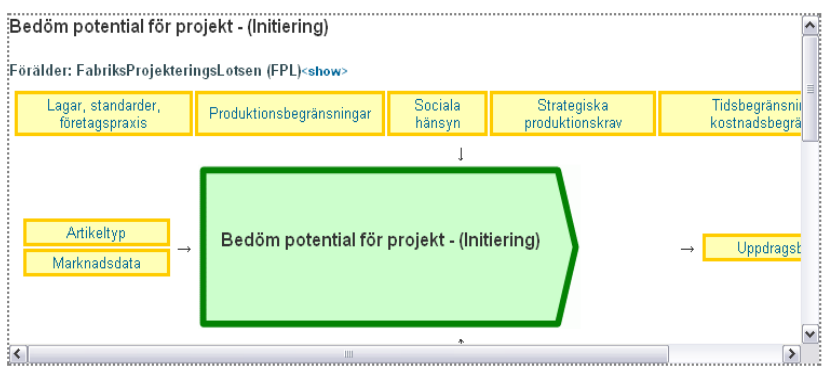

Figure 1: The pilot editing window, a screen-shot [7]

\section{PRODUCTION PILOT}

\subsection{Knowledge system reviews}

There are various kinds of knowledge systems, which can be called a knowledge management system [4], a knowledge reference system or a knowledge-based system [5] [8]. Below, some comparisons are made between the pilot, which is a model-based knowledge reference system, and other knowledge systems. Before comparing, it is important to understand how knowledge is represented and what the thinking behind the pilot is. As already mentioned, knowledge is separated as two levels in the pilot. On one level, the knowledge is represented as an activity model, a computer interpretable knowledge model. The other level is the documented advice in each activity with examples and templates. This makes the knowledge and information more explicitly expressed, although the knowledge and information on this level is not computer interpretable. A part of the activity model that is represented in the pilot is available in Chen [9]. The activities in the model have attributes such as ID, name, and documented advice. Each activity in the model can have one of several relationships to other activities through interaction objects. More details on the interaction objects are presented in a later section. The concept model-based (related to the pilot) means that the model in itself is the knowledge carrier and not the "text files", such as text in word files or pdf files.

Knowledge interpretations can vary depending on the situation, and a computer interpretable form of knowledge is not always the best way for human communication and vice versa. In the pilot the computer interpretable knowledge is used to automatically generate a graphical activity model, which is human cognitive. 
The production pilot vs. an integrated knowledge reference system for product development [10]

In the paper by Bernard et al. the knowledge is analyzed and categorized so that its properties can be written as a vector, placed in a coordinate system [10]. Below is a list of the similarities and the differences compare to the pilot:

Similarities:

- Information and knowledge can be defined and analyzed

- A certain development process can be facilitated

- The knowledge can evolve.

Differences:

- The definitions or descriptions of the knowledge are different. It has been pointed out that the knowledge has different states based on time [10] while the authors in this paper define the different states of the knowledge as data, information, knowledge and experience.

- In the paper by Bernard et al the knowledge is organized as vectors in a coordinate system for product development, while in this paper the knowledge and information is organized into different areas and layers for a better integration and reuse in factory development activities.

- The information and knowledge is more specific in the pilot.

- The knowledge reference system is a theory [10] while the pilot is a product.

The production pilot vs. a knowledge-based system based on a marketing decision model [8]

The knowledge-based system from Yavuz et al is a typical knowledge-based system with a set of decision rules in the system kernel to represent the knowledge. Yavuz el al is using the Prologa system to model their knowledge-based system. The Prologa system itself is a knowledge modeling IT-tool [11] using a decision table.

Similarities:

- Each system, as a product, is an IT application.

- Both systems are information and knowledge driven. The decision model in the paper by Yavuz et al is driven by the decision rules. The pilot is driven by the interaction-object, which poses the information requirement for moving on further in the factory development process.

Differences:

- They are applied to different areas, one is for marketing and the other is for factory development.

- The pilot uses an interface based on SADT and is driven by information in the ICOMs while the other one is using a decision table interface and driven by question rules.

- The pilot has an in-house developed modeling application while Yavuz et al use an existing knowledge modeling system, Prologa, to build their system.

\subsection{Differences between the production pilot and an Al based} knowledge system

It is important to make it clear that there are differences between the pilot and an Al based knowledge system. Two important characteristics in $\mathrm{Al}$ are intelligence and machine learning, and these two characteristics are not associated with the pilot. The pilot represents domain knowledge for the user within factory development, but it doesn't in itself contain any intelligence such as making a proposal for the user depending on the user specific situation [12]. The pilot is not a self-learning system. It can be updated by a human and the core is computer interpretable. The pilot doesn't use any language or tool that can perform the reasoning processes to model information which is also associated to $\mathrm{Al}$ [13] [14] [15] [16].

Generally there are two categories of the knowledge systems beyond the Al based knowledge system. One category is the pure theoretical idea [10] [17] and the other category is knowledge acquired in an IT system [8].

\subsection{Factory development}

Factory development is a very big, complex and capital intensive process, which can be seen as an integration of several processes. Of these, the three main disciplines/areas to be considered are production investment, operation planning and factory design and realization. Below is a short description of each of them.

\section{Production investment}

Production investment has focus on the equipment and its communication with suppliers, in most cases the equipment is machines. The production investment supports machine investment with a quality secured process that leads to investments that meet the production goals and with decreased lead-time from identified problem to equipment running.

\section{Operation/process planning}

The process planning has focus on how a part or product should be manufactured in a machine or a manufacturing system. The process handles e.g. the choice of the right type of process, sequence planning, measurement planning, appropriate fixture design, etc.

\section{Factory planning and realization}

This process has focus on how a factory should be prepared for a manufacturing system or a machine. The process handles the integration of the different domains, such as building construction, media and manufacturing system into a whole factory model, which is then realized.

These three areas are interdependent of each other during the development of the factory. These three processes (activity models) are the core of the production pilot. The activity models are modeled in such a way that they can stand by themselves, just as well as they can interact with each other. This pilot can support different scenarios or cases, and these don't have to be large cases such as "building a new factory". Smaller cases can be operation planning for a new product or buying a new machine tool for a cell.

\subsection{Collaboration characteristics}

It is important to identify the collaboration characteristics in a development process where many areas and large capital are involved. For the factory development there are usually a team of experts of each area involved and this behavior is used to model the pilot. The pilot has characteristics of mass collaboration and traditional collaboration, described in [18], to support factory development due to the reasons below and also illustrated in Figure 2:

- For each of the areas the process is a mass collaboration process

- From a holistic perspective, the pilot is a traditional collaboration system, because of its combination of the different areas. 


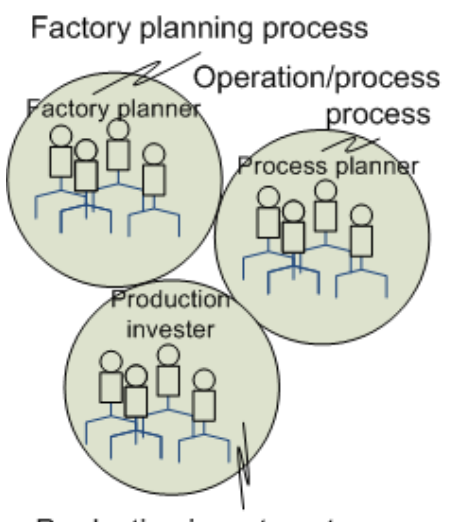

Production investment process Mass
collabaration

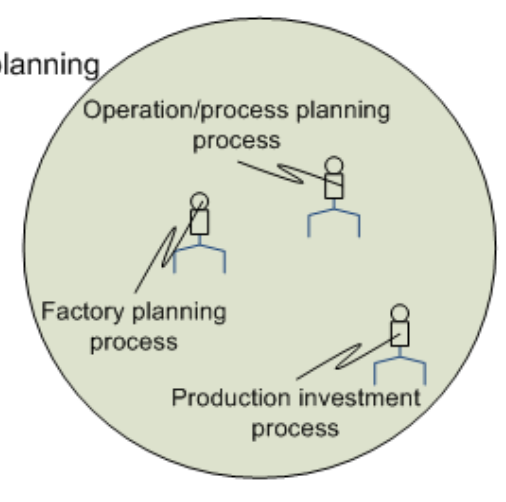

Manufacturing system development process

\section{Traditional collabaration}

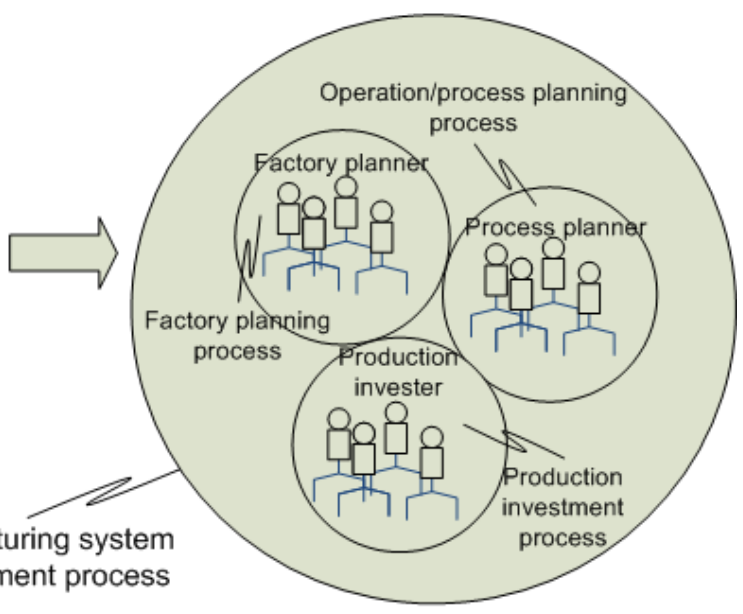

Figure 2: Traditional collaboration combined with mass collaboration during e.g. the manufacturing system development

These characteristics trigger the user of the system to better collaboration, resulting in more structured information, knowledge and a basis for a discussion. This makes the pilot a place to share collected intelligence and knowledge.

\subsection{Interaction between models}

As previously mentioned, this pilot is based on three areas with their activities, which can interact with each other. This interaction is made possible via so called interaction-objects in the pilot, meaning that activities from several areas, or within an area, can share a common object. This interaction-object can also be linked to a terminology library for a better understanding of the concept. The interaction-object uses ICOM objects in SADT modeling. It is also the interaction-object that defines the interface between domains within factory development. It is really important to have the right interaction-objects which are well defined, because they are the links between areas. Figure 3 is a way to illustrate the situation.

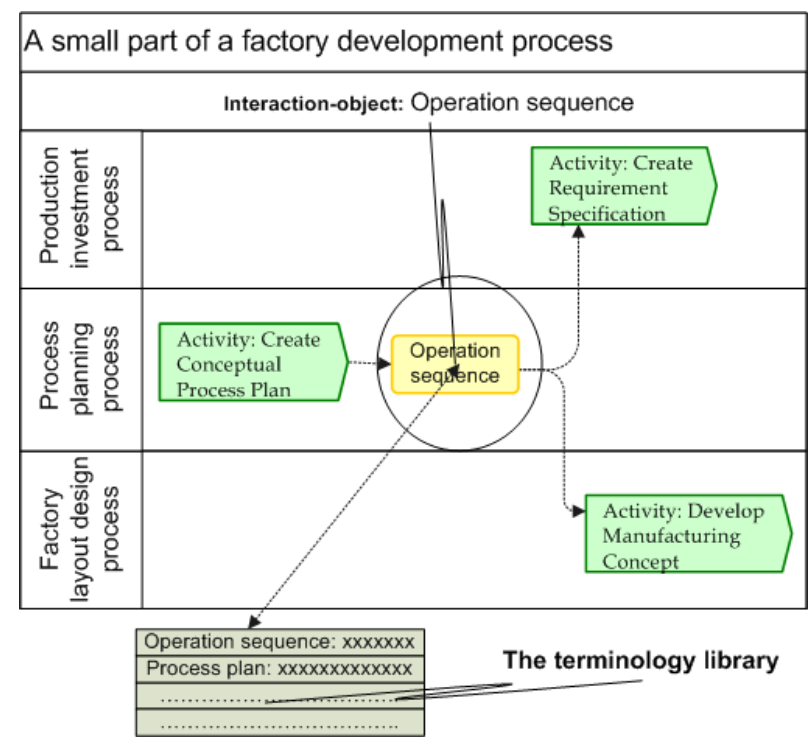

Figure 3: Interaction-object as a link between different activities and terminology

\section{In Figure 3 the interaction-object "Operation sequence" is:}

An output from the activity: Create Conceptual Process Planning.

An input to the activity: Develop Manufacturing Concept.

A mechanism to the activity: Create Requirement Specification.

These three activities are from three different areas in the factory development process, which is described earlier in this paper.

The terminology library is necessary for a uniform understanding of a concept within an area. A typical example of the confusion is the bridge, which can be a game or a bridge-construction. More domain specific examples are the term cycle time and the digital factory, which can be understood or defined differently depending on the users' background [19]

\section{RESULT}

The end result is a production pilot to support co-operation in factory development. This pilot is a way to facilitate the collaboration between disciplines, mostly for the industry, and share and reuse the knowledge and information. The knowledge and information within the production pilot will be reference knowledge for the people in the area and pilot will guide various discipline through a complex process. A detailed list of advantages and limitations follows below.

Some of the advantages of the production pilot:

- Supports different factory development scenarios, overall and in detail. Suited both for small factory-change project as well as building a new factory.

- A part of the knowledge and information is within the activity models and not in external files. This provides a better reuse possibility of knowledge and information i.e., the activity models can be the same but the documented advice, examples and template can be company or project specific.

- The pilot is easy to evolve.

- Has a clear interface between different processes and activities within factory development.

- Contains specific templates and examples for specific activities. 
- $\quad$ Provides a source of knowledge for industrial employees and educational material for both industries and universities in the area of factory development.

- Provides important standards and laws to the right activity.

- Provides the best practice within the area.

- It is an internet-based system which is portable and accessible.

- Is a platform for future research in knowledge sharing.

\section{Some of the limitations of the production pilot:}

- The pilot is best suited for factories with machine tools, mainly because the ModArt research project had its focus on part manufacturing and the companies within the project were mainly machine tool users.

- The standards and laws within the activities are best suited for factories in Sweden, because Swedish laws and standards are used.

- The pilot is in Swedish only.

- The pilot does not cover some interface activities to other domains.

- The best practices and knowledge are collected from few companies.

- $\quad$ Project management is missing.

During the development of the production pilot a big effort has been put into the knowledge representation. It is important to point this out, because many times the focus can be lost and the IT part is taking over.

The structure of the production pilot is illustrated in Figure 4 below, in which the overall level consists of two parts, the knowledge and the modeling application. Further, the knowledge in turn consists of two parts, the documented advice, such as guides, regulations and templates, and the model itself which carries the semantic information, e.g. how the interaction-objects are interconnected.

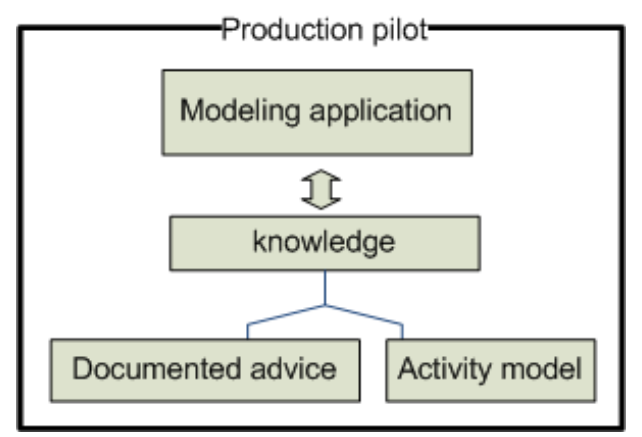

Figure 4: The production pilot structure

There are some other web handbooks that call themselves pilots, e.g. konstruktörslotsen [20]. The pilot has more focus on the knowledge representation and knowledge modeling, compared to konstruktörslotsen, which is a simpler electronic handbook made in HTML.

\section{SUMMARY}

This paper is dedicated to the factory development domain, where knowledge management is a big challenge due to the complexity of its process, which concerns many domains such as building construction, operation planning and machine investment. The challenges derived from knowledge management are collection of knowledge, integration of the knowledge and representation of knowledge for the best understanding and reuse, to support the factory development process in order to avoid the mistakes, shorter down the project time, etc.

This paper presents a production pilot for co-operation in factory development. To realize this pilot:

- A process mapping and knowledge gathering is performed.

- The main domains within factory development are described and important interaction information between these main domains is defined.

- Evaluation between various knowledge systems has been carried out.

- The SADT modeling principle is chosen to model the pilot core.

- An in-house developed modeling application is made to realize this pilot.

- Different collaboration methods are studied.

The pilot has the aim to ease the knowledge reuse and create a value adding working process, for different types of factory development processes. The idea of the modeling application and the user interface is easy access and easy understanding. In support of the easy access, the only software the user needs in order to access and use the modeling application is a basic web browser.

\section{DISCUSSION AND EXTENSION POSSIBILITIES}

There are plenty of extension possibilities of this pilot, thanks to its architecture. The modeling application can be applied to different areas, to create other pilots. The pilot can also be extended to be more company or project specific. Apart from adapting other domains, the extension possibilities of the production pilot itself are huge, such as:

- To link the pilot to the specific IT applications e.g. the applications for the factory layout design, in order to support the factory design in detail. In the factory layout design there are several disciplines involved and the communication is not always clear. The user will then have guidance to move further in each designing step across the disciplines and have a better understanding of the designing background and avoid mistakes.

- To evolve the terminology and interaction-object into ontology to strengthen its consistency. This means that instead of terminology it will be ontology that contains the classification and relation between concepts, and not only the definition of the terms. The name of the interaction-object will be a term in this domain ontology. With domain ontology the reasoning service can also be adopted into the pilot to give proposal for, e.g. the right law and standard for the right situation. It is impossible for a single person to remember all laws and standards during the factory development.

\section{ACKNOWLEDGMENTS}

This work is financed by VINNOVA's MERA and FFI program, and supported by the EXPRES project.

\section{REFERENCES}

[1] Vinnova (2007): ModArt, in: The MERA program projects, pp. 170-176, ISSN 1650-3120. 
[2] Marca, D. A.; McGowan C. L. (1988): Structured Analysis and Design Technique, McGraw-Hill.

[3] Kjellberg, T.; Larsson, M.; Johansson, P.; Wingård, L. (2007): Innovative development of human resources in enterprise and in society - Taxonomy of terms and definitions for competence management, TUT press.

[4] He, W.; Qiao, Q.; Wei, K. (2009): Social relationship and its role in knowledge management systems usage, in: Information and management, Vol. 46, pp. 175-180.

[5] Cochrane, S.; Young, R.; Case, K.; Harding, J.; Gao, J. (2008): Knowledge reuse in manufacturability analysis, in: Robotics and Computer-Integrated Manufacturing. Vol. 25 pp. 508-513.

[6] Hendriks, P.H. (1999): The organizational impact of knowledge-based systems: a knowledge perspective, in: Knowledge-Based Systems, Vol. 12, pp. 159-169.

[7] DMMS (2009): Produktionslotsen, 2010-03-05, www.produktionslotsen.se.

[8] Yavuz, U.; Hasiloglu, A.S.; Kaya, M.D.; Karcioglu, R.; Ersoz, S. (2005): Developing a marketing decision model using a knowledge-based system, in: Knowledge-Based Systems, Vol. 18, pp. 125-129.

[9] Chen D. (2009): Information management for the factory planning process, Licentiate thesis, Stockholm

[10] Bernard, A.; Xu, Y. (2009): An integrated knowledge reference system for product development, in: CIRP Annals Manufacturing Technology, Vol. 58, No 2, pp. 119-122.

[11] Vanthienen, J.; Verhelst, M.; Mues, Ch.; Goedertier, S. Prologa, 2010-09-28, http://www.econ.kuleuven.ac.be/prologa/.

[12] Kumar S.; Singh, R. (2007): A knowledge-based system for selection of progressive die components, in: Journal of Achievements in Materials and Manufacturing Engineering, Vol. 1, No. 1, pp. 85-96

[13] Ruiz-Mezcua, B.; Garcia-Crespo, A.; Lopez-Cuadrado, J.L.; Gonzalez-Carrasco, I (2010): An expert system development tool for non Al experts, in: Expert Systems with Applications, Vol. 38, No.1, pp. 597-609

[14] Wen W.; Chen Y.H.; Chen I.C. (2008): A knowledge-based decision support system for measuring enterprise performance, in: Knowledge Based Systems, Vol. 21. No. 2, pp. $148-163$

[15] Bullinger, H-J.; Warschat, J.; Fischer, D.; (1991): Knowledgebased system for material selection for design with new materials, in: Knowledge-Based Systems, Vol. 4, No. 2, pp. 95-102

[16] Kingston, J., K.C. (1998): Designing knowledge based systems: the CommonKADS design model, in: KnowledgeBased Systems, Vol. 11, No. 5-6, pp. 311-319

[17] Xu, Y.; Bernard, A. (2011): Quantifying the value of knowledge within the context of product development, in: Knowledge-Based Systems, Vol. 24, No. 1, pp. 166-175

[18] Fathianathan, M.; Panchal, J.H.; Nee, A.Y.C. (2009): A platform for facilitating mass collaborative product realization, in: CIRP Annals - Manufacturing Technology, Vol. 58, No. 2, 127-130

[19] Chen, D.; Kjellberg, T. (2009): The Digital Factory and Digital Manufacturing - A Review and Discussion, in: CIRP manufacturing, Grenoble, France
[20] Swerea IVF AB, (2002): Konstruktörslotsen, 2010-09-28, http://lotsen.ivf.se/. 